\title{
TRAVERSES, DELAYS AND FATALITIES AT RAILWAY LEVEL CROSSINGs IN GREAT BRITAIN
}

\author{
Andrew W Evans \\ Imperial College London \\ and \\ Peter Hughes \\ University of Huddersfield
}

May 2019

\begin{abstract}
This paper investigates relationships between traverses, delays and fatalities to road users at railway level crossings in Great Britain. A 'traverse' means a passage across a level crossing by a road user, who may be a pedestrian, cyclist, or occupant of a road vehicle. The paper finds that the road users with the highest fatality rate per traverse are pedestrians at passive crossings. Their rate is about three orders of magnitude higher than that of users with the lowest risk, who are road vehicle occupants at railwaycontrolled crossings. The paper considers the choice between automatic and railway-controlled crossings on public roads. Railway-controlled crossings are widely used in Britain. They are about one order of magnitude safer than automatic crossings, but they impose greater delays on users. A formula is developed to give the overall delay to road users at either type of crossing in terms of the numbers of road users and trains per day, and in terms of the length of time that the crossing must be closed to the road to allow the passage of one train. It is found that automatic level crossings cause substantially less delay than railway-controlled level crossings. The official monetary values of road user delay and of preventing a fatality were used to estimate the valuations of delays and fatalities at hypothetical but representative automatic and railway-controlled crossings. These valuations were then used to explore the effect of replacing representative railway-controlled with automatic crossings or vice-versa. It is found that the valuation of the reduced delays from adopting automatic crossings typically outweighs the valuation of the losses from the increased casualties. However, in practice Britain has chosen to retain a large number of railway-controlled crossings, which implies accepting the delays in return for a good level crossing safety record. Finally, an analysis is carried out to determine the additional risk of typical car and walk journeys that involve traversing a level crossing compared with similar journeys that do not. It is found that the additional risk is small for motor vehicle journeys, but substantial for walk journeys.
\end{abstract}

\section{Keywords}

Railways; safety; accidents; fatalities; level crossings; road users.

(c) 2019. CC-BY-NC-ND 4.0

This is the accepted manuscript of a published paper. The bibliographical reference is:

Evans, Andrew W and Peter Hughes (2019). Traverses, delays and fatalities at railway level crossings in Great Britain. Accident Analysis and Prevention, 129, 66-75.

The digital object identifier is: https://doi.org/10.1016/j.aap.2019.05.006

Centre for Transport Studies

Department of Civil and Environmental Engineering

Imperial College London

London SW7 2AZ

a.evans@imperial.ac.uk
Institute of Railway Research University of Huddersfield

Huddersfield

HD1 3DH

p.hughes@hud.ac.uk 


\section{INTRODUCTION}

This paper investigates the safety and economic performance of railway level crossings (LCs) in Great Britain (GB) for vehicular and pedestrian road users. The paper extends the analysis performed by Evans (2011), which considered long-term trends in fatalities and fatal accidents per crossing by type of crossing and type of user in Great Britain. In that paper risk is measured by fatalities or fatal accidents per crossing per unit time. It would have been useful to take into account the usage of crossings, but no data on usage were available.

Since publication of the earlier studies by Evans, data has been made available by the GB railway infrastructure manager, Network Rail (NR) (2017) which provides a detailed inventory of level crossings in GB. The data include the type of each crossing, and the numbers of crossings or 'traverses' per day made by road vehicles and pedestrians separately, as well as the number of traverses by trains. Data on fatalities at level crossings over a 15 -year period were assembled by the authors. Together these data provide the basis for the study described in this paper which considers the rate of fatalities per traverse.

The literature on level crossing safety contains a number of works that attempt to identify relationships between physical and operational characteristic of level crossings and collision rates. In some cases these works even seek to provide theories for the observed relationships. Examples of these works include $\mathrm{Oh}$ et al. (2006) who found that in Korea "the proximity of crossings to commercial areas ...[is] associated with larger numbers of accidents". Haleem (2016) found that the likelihood of fatalities at private level crossings was influenced by the train speed and the gender of road vehicle drivers. Larue (2016) notes that increased activation time of active warning devices is associated with increased collision risk. Horton (2009) found that more reliable motor vehicles had lower collision rates. Starčević et al. (2016) found that rumble strips on the approach to a level crossing may increase drivers' awareness of level crossings and therefore could have a positive effect on risk reduction.

These findings are meaningful by themselves and provide some insight into potential interventions that a railway or highway authority could undertake to improve safety. However, each of these papers stands alone; what is missing from the literature is a common yardstick by which to compare the relative safety of different level crossings. The contribution of this paper is to make systematic use of the newly-available data on traverses to compare level crossings on the basis of their fatalities per traverse. Normalised comparisons of delays between crossings are also made.

The principal choice among level crossing types for use on public roads is between automatic and railway-controlled crossings. The most common form of automatic crossing in Britain is the automatic half barrier (AHB). The most common form of railway-controlled crossing is the manually-controlled barrier (MCB), some of which are operated locally, and others are operated remotely with Closed Circuit Television (CCTV) used to supervise the crossing. Historically the majority of public road crossings were manually operated by a signaller or crossing-keeper at the site. However, from the 1950s in Europe (MTCA 1957) and from the 1960s in Britain, manually operated crossings began to be replaced by automatic crossings. The aims were to reduce costs by eliminating the operator, reduce delays to road users, and improve safety. The hope of improving safety rested in the reduced scope for human error by railway staff brought about by automation. The differences in operation between automatic and railway-controlled level crossing bring with them an inherent difference in closure time of the level crossings. There is the potential for automatic level crossings to not only reduce human error, but also improve traffic flow. Detailed traverse data make possible a rigorous analysis of the benefit to the road network.

The overall safety risk to road users as a result of level crossings has to be considered in the context of the safety risk inherent in any road travel. The literature is very sparse on this matter, the only prior study found was undertaken for the UK House of Commons (2014). That analysis is repeated here with more recent data.

After a brief look at GB safety performance in relation to Europe in the next paragraph, this paper continues as follows. Section 2 presents the definitions of types of level crossing, types of level 
crossing user, traverses and fatalities, and presents the data on these. Section 3 estimates the fatality risk per traverse for road users by type of user and by type of crossing. Section 4 compares delay and fatality risk to road users at the principal types of level crossings used on public roads. Section 5 compares how level crossing risk for car users and pedestrians compares with the regular road risk. Section 6 summarises the conclusions.

\subsection{UK level crossing safety in context with the rest of Europe}

At the outset it is useful to place the performance of Great Britain in a European context. Basic data on the railway safety performance for each country of Europe, labelled 'Common Safety Indicators' (CSIs) are reported by national authorities annually to the European Union Agency for Railways (ERA), and published by the Agency on line (www.era.europa.eu) and in annual safety reports (ERA 2016).

Table 1 provides a broad overview of the relative safety performance of level crossings in GB and the EU calculated by the authors from data published by the ERA. Compared with the average for the rest of Europe, the United Kingdom (which is Great Britain plus Northern Ireland) had a good record. Table 1 shows that in 2012-2016, the UK had $17 \%$ of the number of LC road user fatalities per capita, $29 \%$ of the number of fatalities per rail route-kilometre, and $37 \%$ of the number of fatalities per level crossing.

Table 1: Fatalities to road and footpath users at level crossings: Europe: 2012-2016

\begin{tabular}{l|rrr}
\hline & $U K$ & $R o E^{*}$ & UK/RoE \\
\hline LC user fatalities per year & 6.400 & 294.600 & \\
LC user fatalities per year per million population & 0.098 & 0.580 & 0.170 \\
LC user fatalities per year per thousand rail route-km & 0.396 & 1.370 & 0.289 \\
LC user fatalities per year per thousand LCs & 1.015 & 2.715 & 0.374 \\
\hline *RoE = All the rest of Europe, that is the EU28 plus Norway plus Switzerland minus UK & \\
\hline \multicolumn{4}{l}{ Source: calculated by authors from Common Safety Indicator data published by the European Agency } \\
for Railways in their Annual Safety Report (ERA 2016)
\end{tabular}

Further European comparisons are outside the scope of this paper, but some may be found in ERA (2016 and 2018). The CSIs are the major source of data for the comparative analysis of level crossing performance between European countries; they include data on the numbers of level crossings by type, and on casualties by type of crossing. However, they do not include data on the numbers of traverses. It is possible that some infrastructure managers assemble such data for internal use, but these are not published.

\section{LEVEL CROSSING CLASSIFICATIONS AND TRAVERSE DATA}

This section describes the data and methods of classification that were used in the analysis. Level crossings are classified in accordance with their method of operation and types of warning devices as described in Section 2.1. In this study, safety at level crossings is measured in terms of fatalities normalised by the number of road users traversing a level crossing: Section 2.2 describes the data that were used for normalisation. Section 2.3 discusses the two categories of road user considered in the analysis: pedestrians and cyclists; and occupants of road vehicles (ORVs). Finally, Section 2.4 describes the data on road user fatalities.

\subsection{Classification of level crossings}

There are many detailed types of level crossing in Britain (and elsewhere), but they may be divided into three principal types: railway-controlled, automatic, and passive.

At railway-controlled crossings, the opening and closing of the crossing to the railway or the road is controlled by a railway signaller or crossing-keeper who is a member of the railway staff. The 
operation of the crossing is almost always interlocked with the railway signalling, so that it is not possible to clear the signals for a train unless and until the crossing is proved closed to road users. Modern railway-controlled crossings have lifting full barriers, either operated by a signaller at the site, or operated remotely and supervised by CCTV. A recent development are crossings with obstacle detectors. On the approach of a train these scan the crossing for obstacles in a similar way to staff scanning the crossing through CCTV. Again, the railway signals cannot be cleared for a train unless and until the obstacle detectors report that the crossing is clear. The principal type of railwaycontrolled crossing in Britain is labelled the MCB.

Automatic crossings are operated by the passage of trains without the intervention of railway staff. When a train approaching the crossing reaches a 'strike-in' point, it triggers the operational cycle: first there is a warning to road users; then the crossing is closed to road users; then the train passes; and finally the crossing opens to the road again. The minimum permissible time in Britain between the train striking-in and its reaching the crossing is 27 seconds (Office of Rail and Road 2011, paragraph 77). The best-known type of automatic crossing is the AHB, which has flashing lights and barriers covering only the approach side of the road. The reason for having only half barriers is to provide an escape route for any vehicle or person already on the crossing when the barriers fall. There are various other types of automatic crossing, including some with lights but no barriers. Some automatic level crossings have been converted from passive crossings by the addition of miniature warning lights for road users. The important difference of automatic from railway-controlled crossings is that they are independent of the railway signals. Almost all crossings on public roads are either AHBs (or variants) or MCBs (or variants).

Passive crossings have no active warnings of the approach of trains, so that the safety of persons or vehicles crossing depends on their own vigilance and behaviour. Sometimes telephones are provided to enable users to phone the railway signaller to check that the line is clear. Passive crossings account for the great majority of level crossings: they are typically used on private roads, on farms, or on footpaths. Many, but not all, are little used; some usage is seasonal or can change with land use.

Some level crossings may be used by both road vehicles and pedestrians, but others are open only to pedestrians (and cyclists). The latter are footpath crossings. All railway-controlled crossings are open to vehicles, but a few automatic crossings and many passive crossings are open only to pedestrians.

\subsection{Road user traverses of level crossings}

A "traverse" is a single passage over a level crossing by a pedestrian, cyclist or a road vehicle. The source of the data on LC traverses is also Network Rail's database of level crossings (Network Rail, 2017). These data include the results of counts, some of which will have been made some years before the nominal date of 2017. NR collect the data on traverses to provide part of the input to their risk assessment model for each level crossing, named the All Level Crossing Risk Model (ALCRM) (Rail Safety and Standards Board, 2007, 2010). Most of their counts of traverses by road users are estimated from counts of users observed over short periods of time and grossed up to a full day. Separate counts are made for pedestrians/cyclists and for motor vehicles. Some of the data for individual crossings are likely to have large standard errors, but this paper assumes that the counts are reasonably accurate when taken over large groups of crossings. Some crossings are described as having "infrequent" use by pedestrians or motor vehicles or both. Such use is assumed for numerical purposes to be 0.5 traverses per day, but the precise assumption does not make much difference to the conclusions. The database was downloaded for this analysis in March 2017. As noted above, these data describe the position nominally as at that date, though the different counts will have been carried out at different dates going back some years into the past. It is noted that traffic volumes may have varied over the 15year period for which fatality data has been collected. This analysis assumes that the degree of variation would be approximately even across all classes of level crossing. Figure 1 plots the percentage of traverses accounted for by each combination of user type and crossing type.

Table 2 gives an overview of the numbers of crossings, both public and private, of each type in Great Britain and the numbers of traverses across them by users. The categorisation of road users and level 
crossings is the same as in the earlier work (Evans, 2011), the source is the NR database (Network Rail, 2017).

It should be noted that NR database contains records of more crossings than the 5863 shown in Table 2. The explanation is that for about 650 crossings the database contains two separate records for what is operationally the same crossing. The duplicates were identified by searching for pairs of crossings with the same geographical coordinates. About 650 pairs were found. Each of these pairs was replaced by a single record with all the necessary information. Almost all the duplicates were crossings where a public footpath coincided with a private road, and crossed the railway at the same crossing. It is understood that there may be legal reasons for retaining two records for them.

Table 2: Numbers of level crossings and traverses per day: Great Britain: March 2017

\begin{tabular}{l|rrrrr}
\hline Crossing type & $\begin{array}{r}\text { No of } \\
\text { crossings }\end{array}$ & $\begin{array}{r}\text { Ped \& Cyc } \\
\text { traverses }\end{array}$ & $\begin{array}{r}\text { Motor Veh } \\
\text { traverses }\end{array}$ & $\begin{array}{r}\text { ORV } \\
\text { traverses }\end{array}$ & $\begin{array}{r}\text { All person } \\
\text { traverses }\end{array}$ \\
\hline Railway-controlled & & & & & \\
Automatic: & 820 & 512,203 & $2,445,996$ & $3,815,754$ & $4,327,957$ \\
$\quad$ Open to vehicles \& peds & 688 & 66,448 & 1,019191 & $1,589,938$ & $1,656,386$ \\
$\quad$ Open to pedestrians only & 60 & 5,330 & 0 & 0 & 5,330 \\
All automatic crossings & 748 & 71,778 & $1,019,191$ & $1,589,938$ & $1,661,716$ \\
& & & & & \\
Passive: & & & & & \\
$\quad$ Open to vehicles \& peds & 2201 & 21,222 & 13235 & 20,647 & 41,869 \\
$\quad$ Open to pedestrians only & 2094 & 47,379 & 0 & 0 & 47,379 \\
$\quad$ All passive crossings & 4295 & 68,601 & 13,235 & 20,647 & 89,248 \\
& & & & & \\
All crossings & 5863 & 652,583 & $3,478,422$ & $5,426,339$ & $6,078,922$ \\
\hline
\end{tabular}

\subsection{Classification of road users}

Traverses of LCs by ORVs are not observed directly because the individuals are inside vehicles, and only road vehicle movements are observed. Vehicle traverses are converted into ORV traverses by multiplying by the average vehicle occupancy, taken to be 1.56. That figure is based on National Travel Survey data.

The right-hand columns of Table 2 give numbers of road user traverses per day by crossing type and by road user type. There are about 6 million person traverses per day in all, of which $11 \%$ are pedestrians or cyclists and $89 \%$ are ORVs. Railway-controlled crossings represent only $14 \%$ of all crossings, but account for $71 \%$ of all person-traverses. Automatic crossings represent $13 \%$ of all crossings and $27 \%$ of all person-traverses. That leaves passive crossings, which are by far the most numerous, representing $73 \%$ of all crossings, but these account for only about $2 \%$ of all persontraverses.

\subsection{Level crossing road user fatalities}

The data on level crossing fatalities cover the 15 calendar years 2003 to 2017. The reason for taking a fairly long period is that $\mathrm{LC}$ fatalities are rare events, and a long period is needed to assemble enough data to observe the accident pattern. The primary source of fatality data was RSSB's Annual Safety Performance Reports (ASPRs). Each successive edition of the ASPR gives details of the fatal LC accidents that occurred in the year to which it refers, including date, location, LC type and road user type. Table 3 gives the fatalities by road user type and by crossing type. There were 131 road or footpath user fatalities in the 15 years, of which 97 were pedestrians or cyclists and 34 were ORVs. There were 12 fatalities at railway-controlled crossings, 46 at automatic crossings, and 73 at passive crossings. This pattern of fatalities is very different from the pattern of traverses given above. 
Table 3: Level crossing road user fatalities by type of user and type of crossing: 2003-2017

\begin{tabular}{l|rrr}
\hline Crossing type & \multicolumn{3}{|c}{ Fatalities 2003-2017 } \\
& $\begin{array}{r}\text { Pedestrian } \\
\text { \& Cycle } \\
\text { Fatalities }\end{array}$ & $\begin{array}{r}\text { ORV } \\
\text { Fatalities }\end{array}$ & Fatalities \\
\hline Railway-controlled & 11 & 1 & 12 \\
Automatic: & & & \\
$\quad$ Open to vehicles \& pedestrians & 18 & 23 & 41 \\
$\quad$ Open to pedestrians only & 5 & 0 & 5 \\
$\quad$ All automatic crossings & 23 & 23 & 46 \\
Passive: & 16 & 10 & 26 \\
$\quad$ Open to vehicles \& pedestrians & 47 & 0 & 47 \\
Open to pedestrians only & 63 & 10 & 73 \\
All passive crossings & & & \\
& 97 & 34 & 131 \\
All crossings & \multicolumn{3}{c}{} \\
\hline
\end{tabular}

It should be noted that in the same period there were also 6 train occupant fatalities, all in the same event. This was the train/car collision and derailment at Ufton Automatic Half Barrier LC in 2004 (RSSB 2005). These fatalities are excluded from the analysis, because this paper is concerned only with road or footpath fatalities.

Furthermore the paper includes only accidental fatalities. Cases where a collision was judged to have been caused by a motivation for self-harm or suicide are excluded from the analysis The exclusions include the fatality to the ORV in the collision above at Ufton. Suicides include fatalities so determined by a coroner, together with suspected suicides assessed according to the so-called "Ovenstone" criteria for assessing suspected suicides in the absence of a coroner's verdict. (See RSSB, 2018, page 151).

Figures 1 and 2 summarise graphically the data from Tables 1 and 2 on traverses and fatalities at level crossing. Figure 1 plots the distribution of traverses among level crossing types and user types and Figure 2 does so for fatalities.

Figure 1: Distribution of person-traverses

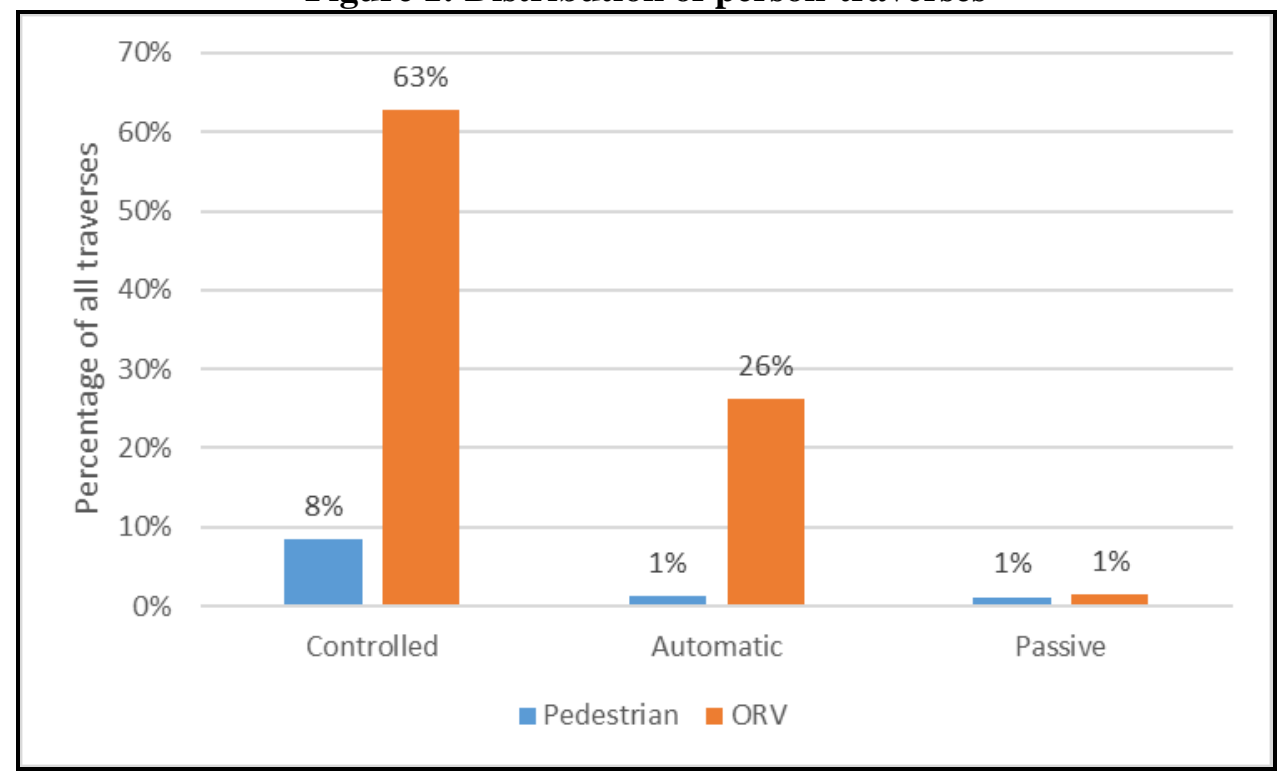


Figure 2: Distribution of fatalities

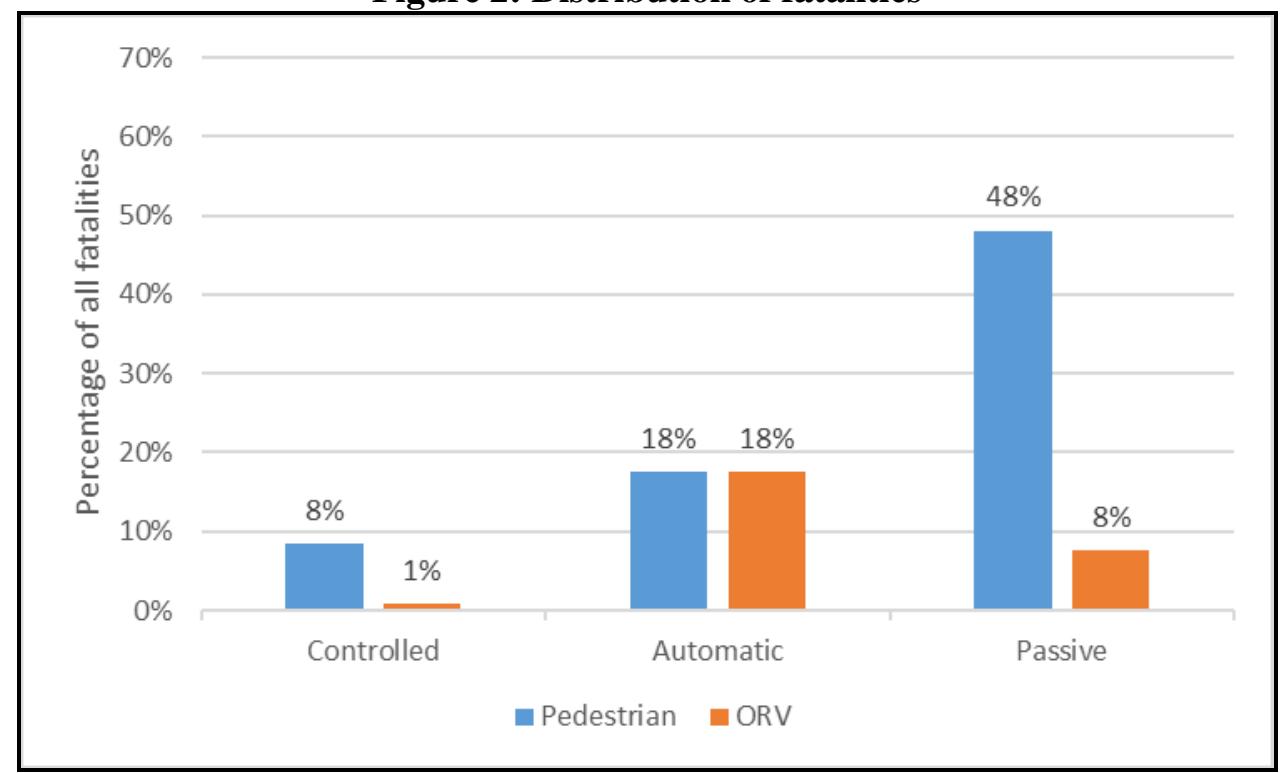

It can be seen from Figures 1 and 2 and from Tables 2 and 3 that the pattern of fatalities among the user types and level crossing types is very different from the pattern of traverses. Specifically, 63 out of 131 fatalities (48\%) occurred to pedestrians at passive crossings, but these account for only $1 \%$ of the traverses. At the opposite end of the scale, only 1 of the 131 fatalities occurred to an ORV at a railway-controlled crossing, but these account for $63 \%$ of the traverses. Such disparities between the distribution of fatalities and that of traverses imply widely differing numbers of fatalities per billion traverses.

\section{ANALSIS OF LEVEL CROSSING FATALITY RISK}

LC fatality risk is taken to be fatalities per billion traverses, averaged over the 15 years 2003-2017, calculated separately for different crossing types and user types. Traverse data are available only for 2017, and investigation is needed to consider whether it is reasonable to allow the 2017 traverse data, suitably scaled, to represent the whole period. It is reasonable to allow this provided that the numbers of traverses do not change greatly over time. Although no data on traverses are available before 2017, data are available on the numbers of crossings by type back to 2003 (and earlier).

Table 4: Level crossings, train-km, road motor vehicle-km and pedestrian-km: 2003 and 2017

\begin{tabular}{l|rrrr} 
& Source & 2003 & 2017 & Change \\
\hline Number of railway-controlled LCs & See below & 864 & 820 & $-5.1 \%$ \\
Number of automatic LCs (all types) & See below & 811 & 748 & $-7.8 \%$ \\
Number of passive vehicular LCs & See below & 3967 & 2201 & $-44.5 \%$ \\
Number of passive pedestrian LCs & See below & 2546 & 2094 & $-17.8 \%$ \\
Total number of LCs & & 8188 & 5863 & $-28.4 \%$ \\
& & & & \\
Train-km (billion per year) & RSSB & 523 & 577 & $+10.3 \%$ \\
Motor vehicle-km (billion per year) & TRA0202 & 487 & 526 & $+8.0 \%$ \\
Pedestrian-km per head per year & NTS0410 & 272 & 272 & $0.0 \%$ \\
\hline
\end{tabular}

\section{Sources}

LC data in 2003: Health and Safety Executive (2004);

Motor-vehicle-km: Department for Transport statistical table TRA0202.

Pedestrian-km: Department for Transport statistical table NTS0410

Network Rail has a policy of closing level crossings when it is practicable to do so, and not to open new ones. The top panel of Table 4 shows the numbers of crossings in 2003 and 2017, and the changes 
between these years. It can be seen that the number of railway-controlled crossings fell by $5 \%$, automatics fell by $8 \%$, passive vehicular crossings by $45 \%$, and passive footpaths by $18 \%$. These changes reflect the relative cost and effort required to close crossings of different types. Railwaycontrolled and automatic crossings are usually on public roads and often busy. Their closure may require replacement by a bridge or an underpass, and perhaps redesign of the local road network. By contrast, passive vehicular crossings are typically on farm roads, and are private, which means that they may have only one or a handful of registered users. Many see little or no use. Many of these crossings may be closed by negotiation between Network Rail and the user(s). However, it is notable that even after the closure of 1,766 passive vehicular crossings between 2003 and 2017, 62\% of the remainder are still described as having "infrequent use". Passive footpath crossings are a mixture of types: some are busy and need to be replaced by bridges or underpasses; others see little use.

The lower panel of Table 4 gives changes between 2003 and 2017 in the quantity of national rail and road use that might be expected to affect the number of level crossing traverses aside from the numbers of crossings. The number of train-kilometres increased by $10 \%$; the number of motor vehiclekilometres increased by $8 \%$; the number of pedestrian-kilometres was unchanged. Given that the number of rail passenger-kilometres increased by $60 \%$ in this period, the $10 \%$ increase in trainkilometres looks modest. There are two explanations. The first is that most of the additional passengerkilometres were carried on the existing services rather than new ones: the average number of passengers per passenger train rose from 91 in 2003 to 126 in 2017. The second explanation is that train-kilometres include freight trains, and these fell between 2003 and 2017.

Thus the effect of level crossing closures between 2003 and 2017 is generally to have reduced the numbers of traverses, and the effect of increased rail and road use is generally to have increased them. For railway-controlled and automatic crossings, the changes are modest as well as working in opposite directions, so it appears reasonable to assume that traverses have remained approximately constant. The falls in the numbers of passive crossings have been much larger, but many of the closed crossings will have had very low usage, so the effect of the closures on traverses will have been modest. Therefore again it seems reasonable to assume that traverses have remained approximately constant.

Table 5 shows observed fatalities per billion traverses. The range is from 0.05 fatalities per billion traverses for ORVs at railway-controlled crossings to 181 for pedestrians at passive footpaths and 171 for pedestrians at the small number of automatic footpaths.

Table 5: Fatalities per billion traverses

\begin{tabular}{l|rrr}
\hline Crossing type & $\begin{array}{r}\text { Pedestrians } \\
\text { \& Cyclists }\end{array}$ & ORV & All \\
\hline Railway-controlled & 3.9 & 0.05 & 0.5 \\
Automatic: & 49.4 & 2.6 & 4.5 \\
$\quad$ Open to vehicles \& peds & 171.2 & & 171.2 \\
$\quad$ Open to pedestrians only & 58.5 & 2.6 & 5.1 \\
$\quad$ All automatic crossings & 137.6 & 88.4 & 113.3 \\
Passive: & 181.1 & & 181.1 \\
$\quad$ Open to vehicles \& peds & 167.6 & 88.4 & 149.3 \\
$\quad$ Open to pedestrians only & & & \\
$\quad$ All passive crossings & 27.1 & 1.1 & 3.9 \\
\hline
\end{tabular}

In order to gain an understanding of the pattern of fatalities in relation to traverses a simple log-linear model was fitted to the data using Poisson regression. Expressed in multiplicative terms, the model takes the following form:

$r_{i j}=s^{*} p_{i}^{*} q_{j}^{*} t_{i j}$ 
where $r_{i j}$ is the number of fatalities per billion traverses suffered by LC users of type $i$ (ORVs or pedestrians) at crossings of type $j$ (railway-controlled, automatic or passive); $p_{i}$ is a multiplying factor for users of type $i ; q_{j}$ is a multiplying factor for crossings of type $j ; s$ is an overall constant of proportionality; and $t_{i j}$ is the number of traverses undertaken by users of type $i$ over crossings of type $j$.

This model assumes that the number of fatalities to each type of user at each type of crossing is proportional to the number of traverses, to a factor related to the type of user, and to a further factor related to the type of crossing.

In expression (1), one of the $p$ 's is arbitrary, because any chosen $p$, say $p_{1}$, can be multiplied by any quantity without altering the value of $r_{i j}$, provided $s$ is divided by the same quantity at the same time. Similarly, one of the $q$ 's is arbitrary. This indeterminacy is here resolved by setting $p_{1}=q_{1}=1$. Table 6 then gives the resulting estimated parameters.

Table 6: Parameters in model for fatalities per billion traverses

\begin{tabular}{l|rr}
\hline Description & Symbol & Value \\
\hline Constant (fatalities per billion traverses) & $s$ & 0.2004 \\
Factor if user is an ORV & $p_{1}$ & 1 \\
Factor if user is a pedestrian & $p_{2}$ & 13.894 \\
Factor if crossing is railway-controlled & $q_{1}$ & 1 \\
Factor if crossing is automatic & $q_{2}$ & 16.198 \\
Factor if crossing is passive & $q_{3}$ & 68.071 \\
\hline
\end{tabular}

To find the estimated fatalities per billion traverses for any specified user type and crossing type, one starts with the constant in Table 6 , and multiplies it by the $p$-factor and the $q$-factor required. Table 7 gives the results, which are also plotted in Figure 3.

Table 7: Estimated fatalities per billion traverses

\begin{tabular}{l|rr}
\hline User type & ORV & Pedestrian \\
\hline Crossing type & & \\
Railway-controlled & 0.2004 & 2.78 \\
Automatic & 3.25 & 45.1 \\
Passive & 13.6 & 189 \\
\hline
\end{tabular}

Figure 3: Fatalities per billion traverses by user type and crossing type

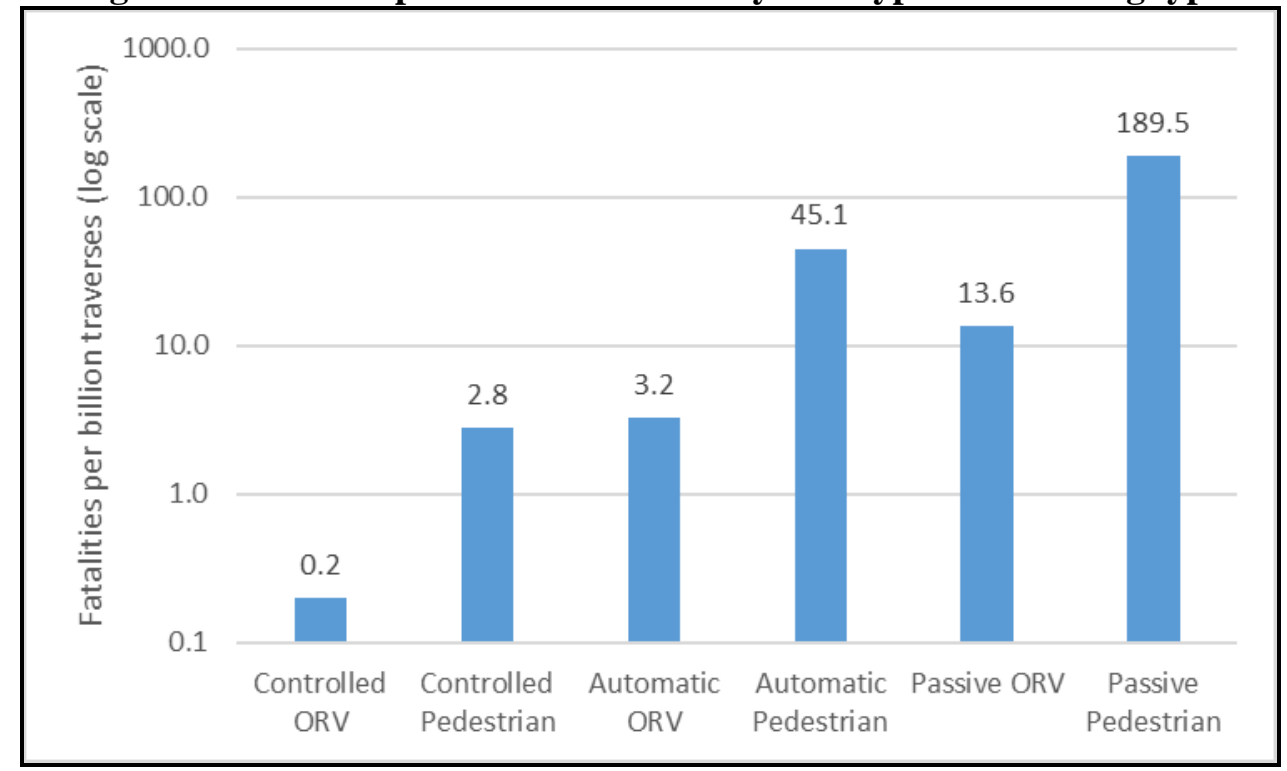


The group with the highest estimated fatality rate per billion traverses is pedestrians at passive crossings. Their estimated fatality rate is about three orders of magnitude greater than that of the group with the lowest rate which is ORVs at railway-controlled crossings. The fatality rates are plotted on a logarithmic scale in Figure 3, which clearly shows the orders of magnitude differences. The rate at passive crossings is about two orders of magnitude greater than that at railway-controlled crossings, and the rate for pedestrians is about one order of magnitude greater than that for ORVs. The rate for automatic crossings is more than one order of magnitude greater than that for railway-controlled crossings, which might be an important consideration in the choice between these two crossing types on public roads.

\section{COMPARISON OF AUTOMATIC AND RAILWAY-CONTROLLED LEVEL CROSSINGS}

\subsection{Simple crossing delays}

The previous section of this paper shows that fatalities per traverse are lower for railway-controlled than for automatic crossings. It follows that the hope that automatic crossings would be safer than manual crossings remains unfulfilled. That is largely because most human error at level crossings is on the part of road users and not railway staff.

On the other hand, automatic crossings generally cause less delay to road users - ORVs and pedestrians - than railway controlled Thus there may be a trade-off between safety and delay in the choice of which crossing type to adopt for particular locations. This choice is illustrated in this section by examining two hypothetical representative level crossings, one with typical properties of railwaycontrolled, but which could be changed to automatic, and the other with typical properties of automatic, but which could be changed to railway-controlled. The representative crossings considered in this section have the average number of traverses by motor vehicles, pedestrians and trains for their type. The operational sequence considered for each crossing is of the most basic type, in which (1) a single train approaches the LC; (2) the LC is closed to the road; (3) the train runs through the LC without stopping; (4) the LC is reopened to the road; and (5) finally the waiting road users clear the crossing. The results for these hypothetical crossings are not necessarily the same as those for any real crossing; the aim of this analysis to not to provide a practical analysis of any real crossing, but to explore by example the structure of the arguments.

Some crossings are substantially more complex than the basic types, involving for example the presence of more than one train in a single road-closure period, or road congestion. These are mentioned again below.

The usage of the representative railway-controlled and automatic crossings is assumed to be the average usage for each type of crossing, calculated from the traverses data in Table 2. Table 8 gives the results. As in Table 2, both directions are taken together. It will be seen that there are about twice as many road user traverses at railway-controlled crossings as at automatics, and about 50\% more train traverses.

Table 8: Traverses at representative crossings

\begin{tabular}{l|rr}
\hline & $\begin{array}{r}\text { Railway- } \\
\text { controlled }\end{array}$ & Automatic \\
\hline RV traverses per day & 2983 & 1481 \\
ORV traverses per day & 4653 & 2311 \\
Pedestrian traverses per day & 625 & 97 \\
All road user traverses per day & 5278 & 2408 \\
Train traverses per day & 97.6 & 66.5 \\
\hline
\end{tabular}

The results on delays are sensitive to the definition of a 'day'. The Network Rail (2017) data give the numbers of traverses made at each crossing in a day. However, neither the road nor the rail flows are likely to be evenly distributed over a 24-hour day. Instead, it is assumed here that both flows are 
concentrated and evenly distributed in an 18-hour day. This point is returned to below. In general, let $b$ be the length of the working day, measured in seconds. If the working day is 18 hours, $b$ is $18 * 3600=$ 64800 seconds.

Figure 4: Delays to road users from the passage of a single train

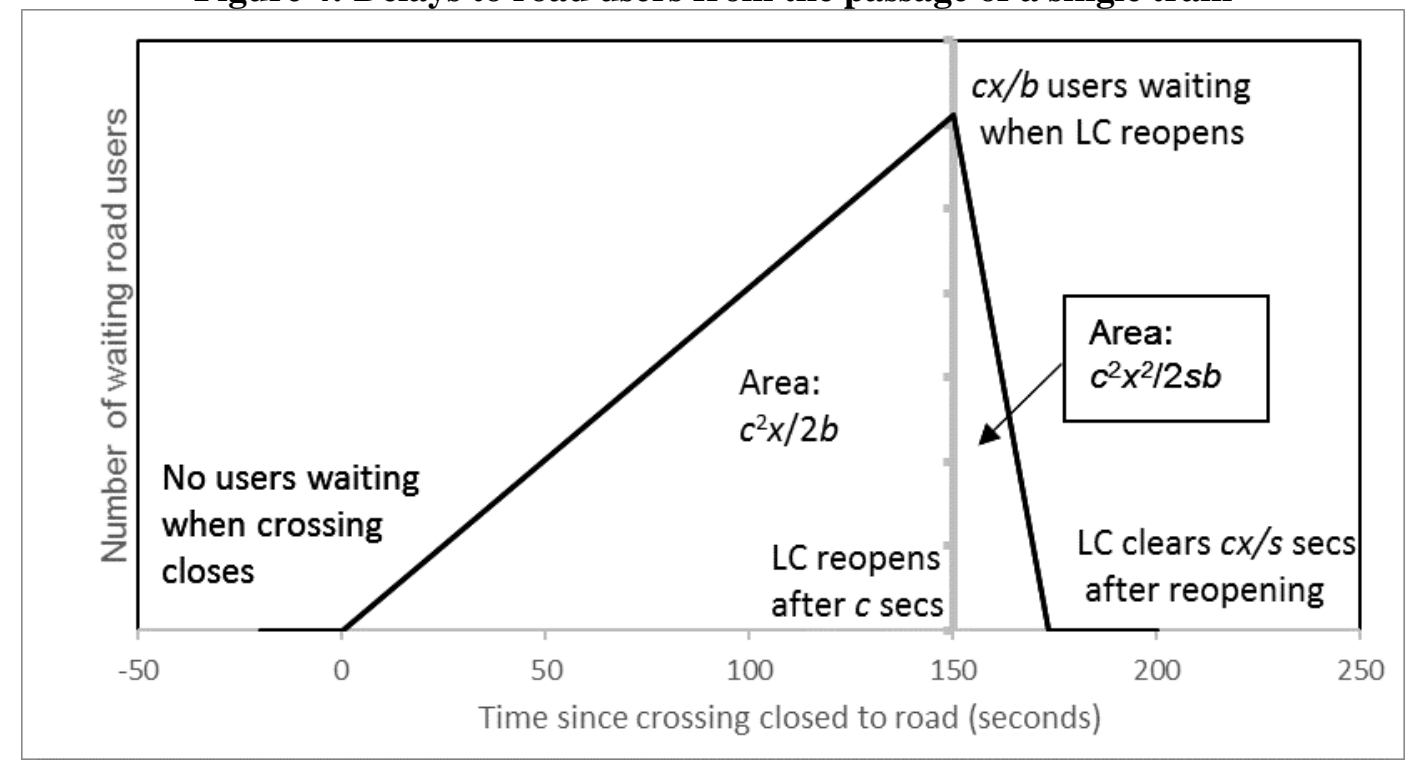

Figure 4 shows the delays to road users resulting from the passage of one train across a level crossing. In this diagram the horizontal axis is time, and the vertical axis is the volume of road users waiting at the crossing. The initial position is shown in the bottom left-hand corner of the diagram, where the crossing is open to the road, and no road users are waiting. The crossing is then closed to the road for the passage of a train, and all arriving road users are delayed. Waiting road users accumulate linearly at the rate of $x / b$ per second, where $x$ is the number of arriving road users per day. After $c$ seconds' closure, the crossing is reopened to the road, and the waiting road users disperse. It is assumed that they depart at the rate of $s$ departures per day, or $s / b$ per second. The number of waiting road users reaches its maximum at the moment when the crossing is reopened, when it is $c x / b$. The number of waiting road users returns to zero when all waiting users have dispersed, which takes place $c x / s$ seconds after reopening. The total delay to road users caused by the passage of the train is the sum of the areas of the two triangles beneath the lines in Figure 4. The area of the left-hand or 'arrival triangle' is $0.5^{*} c^{2} x / b$; the area of the right-hand, or 'dispersal triangle' is $0.5^{*} c^{2} x^{2} / s b$. The area of the dispersal triangle is the fraction $x / s$ of that of the arrival triangle. The area of both triangles together is $0.5^{*} c^{2} x(1+(x / s)) / b$. This delay occurs for each of the $f$ trains per day traversing the crossing. Therefore the delay, $d$, to road users per day is given by:

$d=0.5^{*} c^{2} f x(1+(x / s)) / b$

where $d$ is measured in road-user-seconds per day; $c$ is measured in seconds; $f$ is measured in trains per day; $x$ is measured in road user-traverses per day; $s$ is measured in road users per day; and $b$ is the number of seconds in a working day. Therefore, the total delay to road users using a crossing in a given time is proportional to the product of $f$ and $x$, which is labelled the 'moment' of the rail and road flows, and also proportional to the square of the closure period $c$ for each train. It is not surprising that the delays should be proportional to the moment, but the appearance of the square of $c$ is perhaps less expected. It arises because increasing the closure period $c$ not only increases the number of road users who are delayed, but it also increases the amount of delay. It is this effect that causes long slowmoving trains, such as freight trains, to cause disproportionate delay at level crossings. There is no delay to trains because they are assumed always to be given a clear run. Expression (2) for the delay to road users at active level crossings is simple and elegant. Nevertheless, in a review by RSSB (2007) of 27 level crossing risk models from 12 countries, only one mentions closure periods. 
For practical application, it is convenient to measure delays to road users in terms of road-user-hours per year. Converting from road-user-seconds to road-user-hours requires dividing by 3600 ; converting from delays per day to delays per year requires multiplying by 365 . It is also convenient to replace $b$ by its value of 64800 . With these changes of units. formula (2) becomes

$$
d=0.5 c^{2} f x^{*}(1+(x / s)) *(365) /(3600 * 64800)=7.82322 c^{2} f x^{*}(1+(x / s)) / 10^{7} .
$$

To apply formula (3) for the road user delay at any specified or hypothetical crossing, data are required on train-traverses per working day, $f$, on road-user-traverses per working day, $x$, on the road user dispersal rate, $s$, and on the period of closure to the road, $c$, to allow the passage of one train. As noted above, calculations are made for one representative railway-controlled crossing and one automatic crossing. For these, $f$ and $x$ are assigned their average values given in Table 8 . The value of $s$ is based on the assumption that the road can carry a maximum of 1200 road vehicles per hour, and that road vehicles disperse at that rate. Road vehicles carry 1.56 ORVs, so this vehicular rate implies 1872 ORVs per hour, or 33696 ORVs per day. The maximum flow rate of 1200 vehicles per hour is taken from Rogers (2012, Table 5.13, page 133). A small simplification made is that is it assumed that the dispersal rate for pedestrians is the same as that for ORVs, whereas it may not be, since pedestrians may not need to queue before dispersing. However, this will have little effect on the total delay, because pedestrians make up a small fraction of all road users, and dispersal delay is a small fraction of all delay. The remaining item of data need is $c$, which is discussed in the following section.

\subsection{Period for which level crossings are closed to the road to allow the passage of one train}

The Network Rail (2017) database contains no information on the time for which crossings are closed to the road, $c$, for individual crossings. Nor have the authors identified a paper on this.

However, enough information is available to permit the adoption of reasonably representative values of $c$ for this paper. There are two general sources of information. The first are the rules and standards governing what is required of level crossings. The second are scattered observations. Both sources indicate that closure periods, and therefore road user delay, are much greater for railway-controlled than for automatic crossings.

The safety regulator's requirement for automatic crossings is

"The time between the amber light on each of the road traffic light signals starting to show [the beginning of the cycle] and the train arriving at the crossing should be at least 27 seconds. The train should pass as soon after 27 seconds as possible. At least $95 \%$ of trains should arrive within 75 seconds and 50\% within 50 seconds, once the closing sequence has begun." (ORR 2011, paragraph 77).

The minimum 27 -second approach time would be exceeded by any train travelling at less than the maximum line speed, because these take longer to travel from the strike-in point to the crossing. In addition to the approach time, the crossing remains closed while the train passes over it, and there will be some seconds while the barriers rise. A 200-metre long train travelling at $90 \mathrm{~km} / \mathrm{h}$ would take 8 seconds to pass over a crossing. Therefore, the minimum closure time appears to be about 35 seconds, and with speed variations and a degree of conservatism may be longer. The authors have observed closures periods for single trains at AHBs in the range 32-60 seconds. A representative value of 50 seconds is taken in the main analysis below.

The closure time for railway-controlled crossings is determined by the requirement that the crossing is interlocked with the railway signals. This requires a railway signal capable of showing "stop" (red) or "proceed" (usually green) to be positioned a short distance upstream of the crossing to protect the crossing. (It also requires all equipment to be duplicated for the opposite direction, but that need not be considered here.) The red/ green signal must itself be preceded upstream by a caution signal capable of showing "caution" (yellow or double yellow) or "proceed" (green). If the stop signal is at red, the caution signal must show a caution aspect. The purpose of the caution signal is to warn drivers of 
approaching trains when the stop signal is at red, so that they have adequate time and distance stop at the red signal, using the train's service brake.

Railway-controlled crossings are normally operated so that trains can run through them without slowing down. That requires the caution signal to be at green when sighted by the driver of an approaching train. That in turn requires the stop signal protecting the crossing to be at green, and that requires the crossing to be closed to the road. Therefore, the crossing must be closed to the road from the time an approaching train reaches the sighting point for the caution signal until the train has run through the crossing and the barriers have been raised.

The location of railway signals is determined by the braking requirement of trains and by sighting considerations. RSSB (2015, Appendix A) provides a table giving signalling braking distance data for all trains. The required braking distances depend on the initial speed and on the gradient. For example, the braking distance required from $75 \mathrm{miles} / \mathrm{hr}$ on the level is $1.258 \mathrm{~km}$, and the caution signal must be at least that distance from the stop signal. At that speed that distance takes about 38 seconds. Further time must be added to include the time to cover the sighting distance of the caution signal, and further time to cover the passage of the train through the crossing and the raising of the barriers. This might bring the total to about 70 seconds. However, in practice closure periods at railway-crossings appear to be longer than this analysis suggests. In a brief look at a small number of railway-controlled crossings authors observed a minimum closure period of 98 seconds and a maximum for a single passenger train without a station stop of 225 seconds. A recent digest by the industry (RSSB, 2019, page 6) states that:

"... the time that a [railway-controlled] crossing is closed to the road is generally significantly longer than where an automatic half barrier is provided, and can be as much as three or four minutes for a single train movement."

The explanation is probably that signallers do not wait until the last second before closing the barriers and clearing the signals when trains are approaching, but do so in good time. A representative value of 150 seconds is taken in the main analysis below, three times greater than the corresponding period for AHBs. That may be on the low side.

Given Equation (3), it is simple to calculate the delays for any given level crossing closure periods. As noted above, the main analysis uses 150 seconds for railway-controlled crossings and 50 seconds for automatics. However, the results are also calculated with closure periods one third less, namely 100 seconds and 33 seconds. These are given at the end of this section.

Table 9: Delays to road users at representative railway-controlled and automatic crossings

\begin{tabular}{l|rrrrrr}
\hline Crossing type & $\begin{array}{r}\text { Closure } \\
\text { time for } \\
\text { one train }\end{array}$ & $\begin{array}{r}\text { Train } \\
\text { traverses }\end{array}$ & $\begin{array}{r}\text { Road user } \\
\text { traverses }\end{array}$ & $\begin{array}{r}\text { Arrival delay to } \\
\text { road users }\end{array}$ & $\begin{array}{r}\text { Dispersal delay to } \\
\text { road users }\end{array}$ \\
\hline Units & Seconds & $\begin{array}{r}\text { Traverses } \\
\text { per day }\end{array}$ & $\begin{array}{r}\text { Traverses } \\
\text { per day }\end{array}$ & $\begin{array}{r}\text { Road user hours } \\
\text { per year }\end{array}$ & $\begin{array}{r}\text { Road-user- } \\
\text { hours per year }\end{array}$ \\
\hline Railway controlled & 150 & 97.6 & 5279 & 9065 & 1420 \\
Automatic & 50 & 97.6 & 5279 & 1007 & 158 \\
Automatic & 50 & 66.5 & 2408 & 313 & 22 \\
Railway controlled & 150 & 66.5 & 2408 & 2817 & 201 \\
\hline
\end{tabular}

Table 9 gives the arrival and dispersal delays to road users calculated from Equation (3) and the values of the variables discussed above. The first row of results is for the representative railway-controlled crossing with a closure time of 150 seconds. The second row supposes that that crossing is converted to automatic with a closure time of 50 seconds. It will be seen that both the arrival and the dispersal delays to road users are reduced by a factor of 9 , because the delays are proportional to the square of the closure time. The third row gives results for the representative automatic crossing with a closure time of 50 seconds. The fourth row supposes that that crossing is converted to railway-controlled with a closure time of 150 seconds. That increases the delays by a factor of 9 . 
The results of this paper apply only to simple crossings of the type described in Section 4.1. However, it is possible to make brief comments about more complex situations. One form of complexity is the possible presence of more than one train on a crossing at the same time. This seems generally likely to lead to longer closure times, but it can also lead to smaller delays compared with the simple case. Consider a crossing on a double-track railway with trains approaching in both directions. If the trains are perfectly synchronised so that both reach their respective strike-in points at the same moment and both clear the crossing at the same moment, the closure period will be the same as for one train, namely $c$. That will reduce delays. However, if one of the trains appears $c$ seconds later, so that it strikes in at the same moment as the other train clears the crossing, the closure period for the two trains will be $2 c$. Because the delay is proportional to the square of the closure period, the delay will be double that caused by two separate trains.

Another source of complexity is congestion. It is assumed in the preceding analysis that waiting road users always clear the crossing when it is opened after a train, and none has to wait for another cycle. That will not always be so, in which case delays become longer. However, the data suggest that most crossings have adequate capacity. Table 10 shows how the time in an 18-hour day is allocated between being open to the railway, used by road vehicles (assuming 3 seconds per vehicle), and not used. For the representative railway controlled and automatic crossings, the data suggest that $64 \%$ and $88 \%$ respectively of the time is not used. Of course spare time is useless when and where it is unwanted, and peak hour road flows are typically twice average flows (Department for Transport statistical table TRA0307), but the data suggest that these can generally be accommodated. It should be said that this simple calculation disregards pedestrians.

Table 10: Use of available time at representative crossings

\begin{tabular}{l|rr}
\hline & $\begin{array}{r}\text { Railway- } \\
\text { controlled }\end{array}$ & Automatic \\
\hline Open to railway & $22.6 \%$ & $5.1 \%$ \\
Used by road vehicles & $13.8 \%$ & $6.9 \%$ \\
Not used (by subtraction) & $63.6 \%$ & $88.0 \%$ \\
\hline All & $100.0 \%$ & $100.0 \%$ \\
\hline
\end{tabular}

\subsection{Delays and fatalities at automatic and railway-controlled level crossings}

This section puts together the results on fatalities and delays, and explores the effects of changing the representative railway-controlled crossing to automatic, and of changing the representative automatic crossing to railway controlled. As previously noted, railway-controlled crossings have lower fatality rates than automatic, and in the analysis which follows this difference in rates is attributed to the differences in the design and operation of the crossings and not to possible other causes. In that case switching towards automatic crossings will increase fatality risk but reduce delays, and switching towards railway-controlled will have the opposite effects.

Delays and fatalities are valued and compared using the valuations of avoiding the delay and of preventing the fatalities in £million per year, using the valuations of travel time used by the Department for Transport (2017) in its economic appraisal of transport projects. The valuations used are $£ 7.05$ per hour of travel time, and $£ 1,548,105$ for a prevented fatality, both at 2010 prices 


\begin{tabular}{|c|c|c|c|}
\hline Crossing type & $\begin{array}{l}\text { Closure } \\
\text { time for } \\
\text { one train }\end{array}$ & $\begin{array}{r}\text { Fatalities per } \\
\text { year }\end{array}$ & $\begin{array}{r}\text { Delay per } \\
\text { year }\end{array}$ \\
\hline Units & Seconds & & $\begin{array}{r}\text { Road user } \\
\text { hours per } \\
\text { year }\end{array}$ \\
\hline $\begin{array}{l}\text { Railway controlled } \\
\text { Automatic } \\
\text { Automatic } \\
\text { Railway controlled }\end{array}$ & $\begin{array}{r}150 \\
50 \\
50 \\
150\end{array}$ & $\begin{array}{r}0.975^{*} 10^{-3} \\
15.792^{*} 10^{-3} \\
4.327^{\star} 10^{-3} \\
0.267^{*} 10^{-3}\end{array}$ & $\begin{array}{r}10485 \\
1165 \\
335 \\
3019\end{array}$ \\
\hline
\end{tabular}

Table 11 gives fatalities and delays per year for the same four crossings as in Table 9. The first row gives results for the representative railway-controlled crossing with a closure time of 150 seconds. The second row supposes that that crossing is converted to automatic with a closure time of 50 seconds. The third row gives results for the representative automatic crossing with a closure time of 50 seconds. The fourth row supposes that that crossing is converted to railway-controlled with a closure time of 150 seconds. The fatalities per year in Table 9 are obtained by multiplying the fatalities per traverse in Table 7 for ORVs and pedestrians by the numbers of traverses per year calculated from Table 8 . The delays are the sum of the arrival delay and the dispersal delay given in Table 9.

Table 12 gives valuations of fatalities and delays for the representative crossings. The top panel gives the valuations for the crossings in their assumed initial state. The middle panel supposes that the initial railway-controlled crossing is converted to automatic and the initial automatic crossing is converted to railway-controlled. Changing the type of crossing affects both fatalities and delays: the former because fatality rates are different for different types of crossing; the latter because closure times change. The bottom panel of Table 12 gives these changes. Figure 5 plots the values of these changes.

Table 12: Values of road user delays and fatalities at representative level crossings

\begin{tabular}{l|rr}
\hline & $\begin{array}{r}\text { Currently } \\
\text { railway- } \\
\text { controlled }\end{array}$ & $\begin{array}{r}\text { Currently } \\
\text { Automatic }\end{array}$ \\
\hline Units & £ per year & £ per year \\
\hline Road user fatality rates at representative LCs & 1509 & 6698 \\
Current value of fatalities & 73919 & 2365 \\
Current value of delay & 75429 & 9063 \\
Current value of fatalities and delay & & \\
\hline If current LC type were changed to the opposite & 24447 & 414 \\
Changed value of fatalities & 8213 & 21282 \\
Changed value of delay & 32661 & 21696 \\
Changed value of fatalities and delay & \multicolumn{2}{|r}{} \\
\hline Difference (changed minus original) & 22938 & -6284 \\
Value of fatalities per year) & -65706 & 18918 \\
Value of delays & -42768 & 12633 \\
Value of fatalities and delay & \multicolumn{2}{|c}{} \\
\hline
\end{tabular}

Table 12 and Figure 5 shows that for the currently railway-controlled crossing the value of fatalities is very low at about $£ 1500$ per year, but the value of delays is high at about $£ 74,000$ per year. If that crossing were converted to automatic, the value of fatalities would rise by $£ 23,000$ per year, but the value of delays would fall by $£ 66,000$ per year. Thus the value of the reduction in delay outweighs the loss from the increase in fatalities. This is shown graphically on the left-hand side of Figure 5. It should be noted that the orientation of the vertical axis in Figure 5 is so as to show welfare gains in the positive direction, even though the variable itself may be negative. For example, the reduction in delay of $£ 65,706$ per year appears in the positive direction because reductions in delay are beneficial. 
For the currently automatic crossing, the value of fatalities is about $£ 6700$ per year. This would fall by $£ 6300$ to only $£ 400$ per year if the crossing were converted to railway-controlled. At the same time the value of delays would increase from $£ 2000$ to $£ 20,000$ per year, an increase of $£ 18,000$ per year. The value of the increased delay would outweigh that of the reduced fatalities.

It should be noted that there are other safety benefits besides the prevention of fatalities, notably the prevention of injuries. However, because level crossing accidents tend to be severe when they occur, the value of non-fatal injuries is small relative to the value of fatalities: it was about $10 \%$ in the five years to 2017 (RSSB, 2017). The valuation of injuries is disregarded here.

Figure 5: Changes in welfare if types of level crossings were changed Closure times 150 and 50 seconds

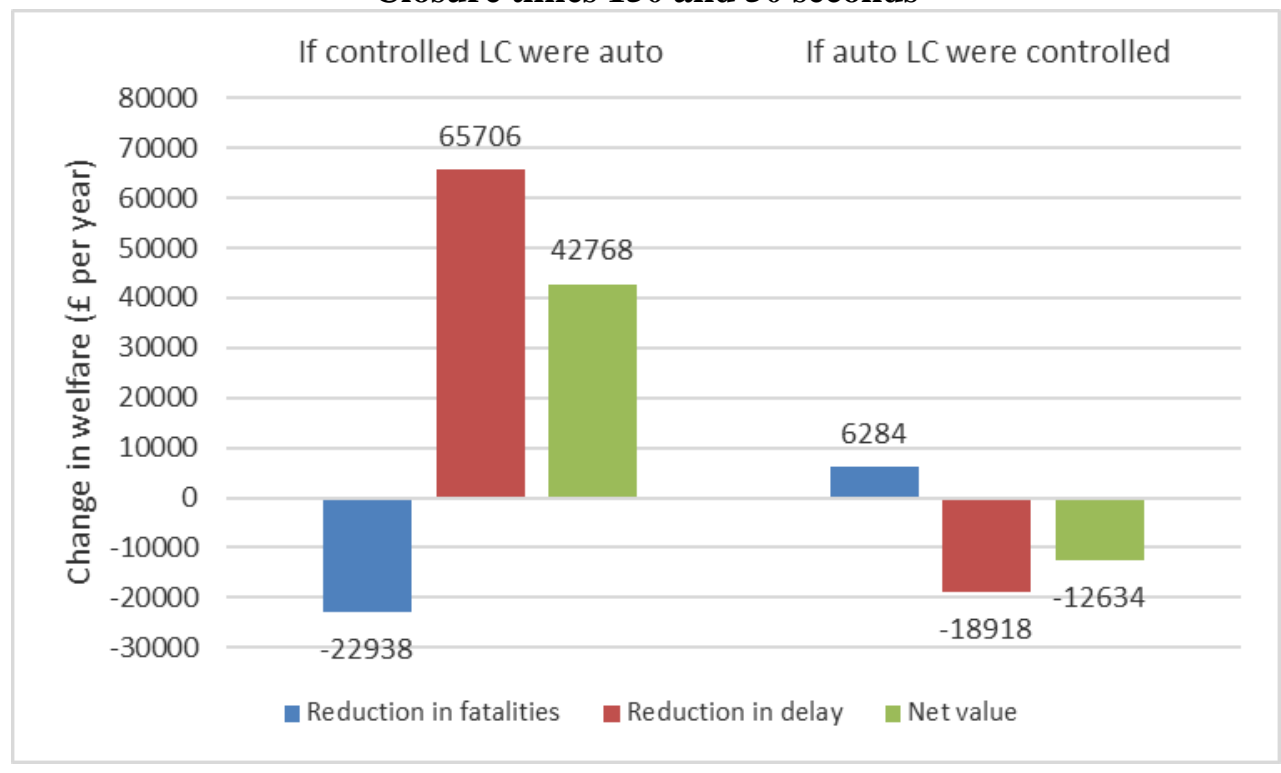

Figure 6: Changes in welfare if types of level crossings were changed Closure times 100 and 33 seconds

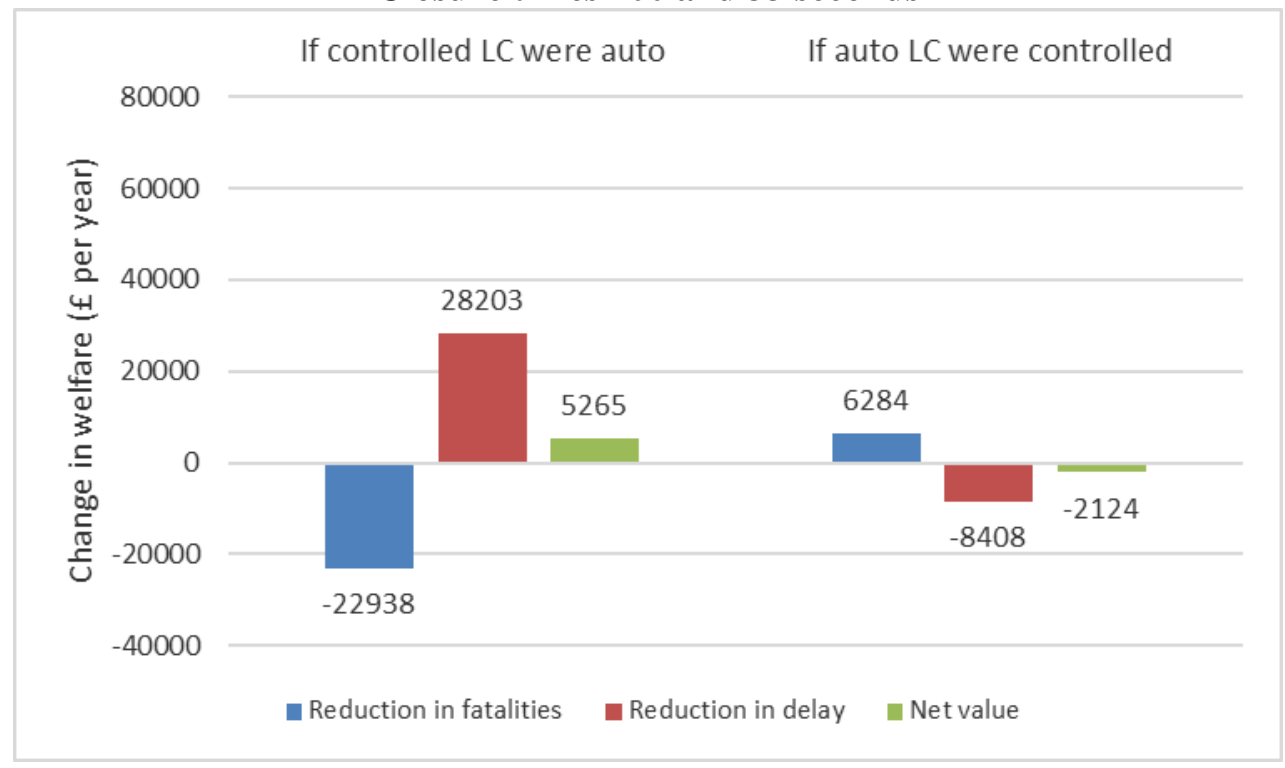


Figure 6 repeats Figure 5, but with closure times of 100 seconds for railway-controlled crossings and 33 seconds for automatics. The qualitative pattern of the results is the same as for the longer closure periods, but the delays are much smaller.

The general conclusion from the representative crossings is that, based on the economic values used in this study, it is possible to increase net economic benefit by converting the railway-controlled crossing to automatic, but this would come at the cost of increased fatalities. It is also possible to decrease fatalities by converting the automatic crossing to railway controlled, but the value of the reduction in fatalities may be outweighed by the value of the increased delay. This points to a genuine trade-off between safety and delay. It is not surprising that different countries make this trade-off in different ways. The importance of the length of closure periods emerges clearly from this analysis. The next generation of level crossings using obstacle detection may be able to combine the high level of safety currently being achieved by railway-controlled crossings with something nearer the speed of automatic crossings.

\section{EFFECT OF PRESENCE OF A LEVEL CROSSING ON TYPICAL CAR AND WALK JOURNEYS}

This section is based on the report of the UK House of Commons Transport Committee for their enquiry on the safety of level crossings in 2014 (UK House of Commons Transport Committee, 2014). An earlier version of Table 13 was published in their report.

In order to place the risk at level crossings in perspective, it is desirable to find a means of comparing LC risks with road risk. One way of doing this would be first to estimate the road safety fatality risk of a typical car trip or walking trip without a level crossing, to form a baseline. Then it could be supposed that the journey required the traverse of one level crossing, and the additional risk imposed by the level crossing could be compared with the baseline risk.

This calculation can be done for both typical car journeys and typical walk journeys, though various simplifications have to be made. Table 13 shows the data used and the method of calculation. The column headed "source" gives either the external source of the data or a calculation from other figures in the table. The alphanumeric sources such as NTS0409 are references to Department for Transport statistical tables. Other references provide the method of calculation based on other data in the table, for example item (c), which reads (b)/(a) is the value obtained by dividing the data at row (b) by the value at row (a).

The bottom line of the table shows the result of the calculation, being the additional risk from the presence of one level crossing on an average car or walk journey as a percent of the baseline road risk. For car journeys, the additional risk from the level crossing is estimated at $8 \%$ of the baseline road risk, which is modest. For walk journeys, the additional risk from the presence of a level crossing is much larger. It is estimated to be $115 \%$ of the baseline road risk, implying that the LC roughly doubles the risk of the journey. However, average walk journeys are shorter than car journeys, so the walker is less likely to encounter a level crossing. 
Table 13: Journeys and fatalities with car and walk as main mode: 2015

\begin{tabular}{|c|c|c|c|}
\hline & Source & Car & Walk \\
\hline $\begin{array}{l}\text { Road journeys } \\
\text { (a) Trips/person/year with given main mode } \\
\text { (b) Km/person/year with given main mode } \\
\text { (c) Average length of trip with given main mode } \\
\text { (km) }\end{array}$ & $\begin{array}{l}\text { NTS0409 } \\
\text { NTS0410 } \\
\text { (b)/(a) }\end{array}$ & $\begin{array}{r}591 \\
8143 \\
13.78\end{array}$ & $\begin{array}{r}243 \\
261 \\
1.073\end{array}$ \\
\hline $\begin{array}{l}\text { Road fatalities } \\
\text { (d) Road fatalities per billion person-km } \\
\text { (e) Road fatalities per billion trips } \\
\text { (f) Road fatalities per single trip }\end{array}$ & $\begin{array}{l}\text { RAS53001 } \\
(c)^{*}(d) \\
(e)^{*} 1 E-09\end{array}$ & $\begin{array}{r}1.1 \\
15.16 \\
1.52 \mathrm{E}-08\end{array}$ & $\begin{array}{r}22 \\
23.60 \\
2.36 \mathrm{E}-08 \\
\end{array}$ \\
\hline $\begin{array}{l}\text { Level crossing traverses } \\
\text { (g) LC vehicle or pedestrian traverses per year } \\
\text { (h) Average car/van occupancy } \\
\text { (i) LC person-traverses per year. }\end{array}$ & $\begin{array}{l}\text { NR LC database } \\
\text { NTS0905 } \\
(\mathrm{g})^{*}(\mathrm{~h})\end{array}$ & $\begin{array}{r}12.69 \mathrm{E}+08 \\
1.56 \\
19.81 \mathrm{E}+08\end{array}$ & $\begin{array}{l}2.38 \mathrm{E}+08 \\
2.38 \mathrm{E}+08\end{array}$ \\
\hline $\begin{array}{l}\text { Level crossing fatalities } \\
\text { (j) LC Fatalities per year 2003-2017 } \\
\text { (k) LC fatalities per person-traverse }\end{array}$ & $\begin{array}{l}\text { RSSB ASPR } \\
\text { (j)/(i) }\end{array}$ & $\begin{array}{r}2.3 \\
1.14 \mathrm{E}-09\end{array}$ & $\begin{array}{r}6.5 \\
2.71 E-08\end{array}$ \\
\hline $\begin{array}{l}\text { LC/road fatalities comparison } \\
\text { (I) LC as percent of road fatalities per trip }\end{array}$ & $(\mathrm{k}) /(\mathrm{f})$ & $8 \%$ & $115 \%$ \\
\hline
\end{tabular}




\section{CONCLUSIONS}

This paper extends the work of Evans (2011) by using data on road user traverses to produce normalised fatality rates at level crossings. The work is significant as it enables future analyses and comparisons of level crossing safety to be based on normalised data.

The work finds that the fatality rate per person-traverse is about one order of magnitude higher at automatic than at railway-controlled crossings, and a further one order of magnitude higher at passive crossings. Cross-cutting this, the fatality rate for pedestrians and cyclists is one order of magnitude higher than that for occupants of road vehicles. The combination of these two factors means that the highest fatality rate, for pedestrians and cyclists at passive crossings, is three orders of magnitude higher than the lowest rate, for occupants of road vehicles at railway-controlled crossings.

The paper investigates the choice between railway-controlled and automatic crossings for public roads. Railway-controlled crossings are safer, but they cause more delay to road users. The paper derives a new expression for the delay, showing that it is proportional to the product of road-user-traverses per day and the number of trains per day (the so-called road user/train moment). The delay to road users is also proportional to the square of the length of time that the crossing is closed to the road for the passage of a train. This time is longer for railway-controlled than automatic crossings because railwaycontrolled crossings, including those monitored locally as well as those monitored remotely by CCTV, are interlocked with the railway signals. This is the source of the greater safety, but it means that the delay for road users is also greater.

The paper explores this relationship between safety and delay by analysing two hypothetical but representative level crossings, one railway-controlled and one automatic. Each representative crossing is presumed to have the average number of train and road user traverses for its type. The paper estimates the changes in fatalities and delays that would arise first if the representative railwaycontrolled crossing were converted to automatic (thereby worsening safety but reducing delay), and secondly if the automatic crossing was converted to railway-controlled (thereby improving safety but increasing delay). Using standard valuations of travel time and for the prevention of fatalities, the general finding is that the value of the time losses from moving in the direction of more railwaycontrolled crossings outweighs the value of the improved safety. However, the British stakeholders have indicated their preferences for safety by opting to retain a large number of safer railwaycontrolled crossings at the expense of greater delays.

The paper concludes by using the newly available traverse data to repeat the calculation performed for the UK House of Commons to calculate the extra fatality risk imposed by the presence of a level crossing on typical car and walk journeys. The additional risk is small for motor vehicle journeys, but substantial for walk journeys.

The existing literature on level crossing safety is characterised by ad-hoc studies without a common framework for comparison of the results. The work described in this paper uses the newly available traverse data as part of a rigorous analysis to provide a solid basis for comparison of level crossing safety. 


\section{REFERENCES}

Department for Transport (2017). WebTAG databook .

European Union Agency for Railways (2016). Railway Safety Performance in the European Union 2017. ERA, Valenciennes.

Evans, A W (2011). Fatal accidents at railway level crossings in Great Britain 1946-2009. Accident Analysis and Prevention 43, 1837-1845.

Haleem, K. (2016). Investigating risk factors of traffic casualties at private highway-railroad grade crossings in the United States. Accident Analysis \& Prevention, 95, 274-283.

Health and Safety Executive (2004). HSE's annual report on railway safety. HSE, London

Horton, S. (2009). Success factors in the reduction of highway-rail grade crossing incidents. US Department of Transportation, Research and Innovative Technology Administration, Volpe National Transportation Systems Center, Cambridge, MA USA.

Larue, G. (2016); Waiting Times at Level Crossings Leading to Motorists' Risky Behaviours; Australasian Centre For Rail Innovation

Ministry of Transport and Civil Aviation (1957). Level Crossing Protection. A report by officers of the Ministry of Transport and Civil Aviation and of the British Transport Commission. HMSO, London.

Network Rail (2017). Level Crossings Correct to Monday 28 December 2016. Retrieved from http://www.networkrail.co.uk/transparency/level-crossings/ 15 March 2017

Office of Rail and Road (2011). Level crossings: a guide for managers, designers and operators. Railway publication 7, ORR London.

Oh, J., Simon P. Washington, and Doohee N, (2006) Accident prediction model for railwayhighway interfaces. Accident Analysis \& Prevention 38.2: 346-356.

Rail Safety and Standards Board (2005). Formal Inquiry Report. Ufton Level Crossing: passenger train collision with a road vehicle and subsequent derailment on 6 November 2004.

Rail Safety and Standards Board (2010). Development of the All Level Crossing Risk Model: A History, 1993-2010. RSSB, 2010.

Rail Safety and Standards Board (2015). Guidance Note GK/GN0675. Lineside Signal Spacing and Speed Signage Issue 4. RSSB, London.

Rail Safety and Standards Board (2017). Annual Safety Performance Report 2016/2017. RSSB, London.

Rail Safety and Standards Board (2018). Annual Safety Performance Report 2017/2018. RSSB, London.

Rail Safety and Standards Board (2019). Level Crossing Diggest V1. RSSB, London.C London Rail Safety and Standards Board (annual). Annual Safety Performance Report. RSSB, London.

Rogers, Martin (2012). Highway Engineering, Blackwell.

RSSB (2007). Use of risk models and risk assessments for level crossings by other railways. RSSB's Research and Development. Report T524, RSSB, London.

Starčević, M., Barić, D., \& Pilko, H. (2016). Survey-based Impact of Influencing Parameters on Level Crossings Safety. Promet-Traffic \&Transportation, 28(6), 639-649.

UK House of Commons Transport Committee (2014). Safety at Level Crossings. Eleventh report of session 2013-2014. 


\begin{tabular}{l|l}
\multicolumn{2}{l}{ ABBREVIATIONS } \\
\hline AHB & Automatic half barrier (level crossing) \\
ALCRM & All Level Crossing Risk Model \\
ASPR & Annual Safety Performance Report \\
CCTV & Closed circuit television \\
CSI & Common Safety Indicator \\
ERA & European Union Agency for Railways \\
EU & European Union \\
GB & Great Britain: England, Wales and Scotland \\
HSE & Health and Safety Executive \\
LC & Level Crossing \\
MCB & Manually-controlled barrier (level crossing) \\
NR & Network Rail (Infrastructure Manager) \\
NTS & National Travel Survey \\
ORR & Office of Rail and Road or (previously) Office of Rail Regulation \\
ORV & Occupants of road vehicles \\
RoE & Rest of Europe: EU28 plus Norway plus Switzerland minus UK. \\
RSSB & Rail Safety and Standards Board \\
UK & United Kingdom: Great Britain and Northern Ireland \\
\hline
\end{tabular}

